\title{
Autoridad, reconocimiento y emancipación Educación de personas en situación de discapacidad
}

Authority, recognition and emancipation: The education of people with disabilities (c)
Uj

IBEROAMERICANA CORPORACIÓN UNIVERSITARIA
HORIZONTES

PEDAGÓGICOS

ISSN-I: $0123-\left.8264\right|^{\text {e-ISSN: } 2500-705 X}$ 


\section{HORIZONTES PEDAGÓGICOS}

ID:

0123-8264.hop.19202

Title: Authority, recognition and emancipation

Subtitle: The education of people with disabilities

Título: $\quad$ Autoridad, reconocimiento y emancipación

Subtítulo: Educación de personas en situación de discapacidad

Alt Title / Título alternativo:

[en]: $\quad$ Authority, recognition and emancipation in the education of people with disabilities

[es]:

Autoridad, reconocimiento y emancipación en la educación de personas con discapacidad

Author (s) / Autor (es):

Schewe

Keywords / Palabras Clave:

[en]:

authority; inclusion; disability; middle education and special school

[es]: $\quad$ autoridad; inclusión; discapacidad; educación media; escuela especial

Proyecto / Project:

Derivado del Seminario Doctoral Autoridad educativa y procesos de emancipación dictado por la Dra. Beatriz Greco, en CONACIT, es parte de la tesis para obtener el título de doctora en educación de la Universidad Nacional de la Plata, Argentina

Submited: 2017-01-31

Acepted: 2017-07-16

\section{Resumen}

La educación inició un período de transición en el año 2006, cuando las leyes internacionales comenzaron a proponer a "la inclusión como el principio fundamental del derecho a la educación". En Argentina,

se intentaron cambios institucionales promovidos desde las políticas públicas. Específicamente, éstas empujaron la creación de nuevos estamentos y espacios de trabajo para que las personas con discapacidad, pudieran asistir a las escuelas de educación común, teniendo la posibilidad de finalizar sus estudios primarios, secundarios y superiores.

La asignación de recursos necesarios para la implementación de los nuevos lineamientos no es suficiente y las condiciones institucionales no están dadas para atender al número de estudiantes que se encuentran formando parte de las aulas de las escuelas de educación común. En el artículo se analiza la cuestión de la autoridad, como parte de estos complejos procesos.

\section{Abstract}

A period of transition in Education began in 2006, when the international laws began to suggest: "the fundamental principles of inclusion to ensure the right of education." In Argentina, through public policies institutional changes were promoted. Particularly, these

pushed the creation of new bodies and workspaces so that people with disabilities could attend regular education schools.

Thus, having the possibility to finish their primary, secondary and higher education. The allocation of necessary resources for the implementation of the new guidelines has not been sufficient and the institutional conditions

have not been enough to meet the needs

of the number of students in classrooms of

regular education schools. The inquiry of this research is to analyze the question of authority, as part of these complex processes, which are suggesting inclusion as a fundamental principle of the right to education.
Lic Carolina Lelia Schewe, [Dr] MSc sp

ORCID: $\quad$ 0000-0003-3773-8909

Source I Filiacion:

Universidad Nacional de Misiones

BIO:

Doctoranda en Ciencias de la Educación, Universidad de La Plata. Becaria de CONICET [ar]

Maestrante en Ciencias Sociales, Universidad Nacional de Quilmes

(Trabajo de tesis en evaluación)

Especialista en Didáctica y Currículum

Profesora en Educación Especial, Universidad Nacional de Misiones

Docente Jefa de Trabajos Prácticos de las asignaturas Pedagogía General, Introducción a la

Educación Especial y Seminario de Investigación Educativa del Profesorado en Educación

Especial de la Universidad Nacional de Misiones

Colaboradora del Observatorio de la Discapacidad, Departamento de Ciencias Sociales,

Universidad Nacional de Quilmes

Docente invitada de la Universidad Católica Nuestra Señora de la Asunción de Itapúa (Paraguay) Investigadora Categoría IV del Programa Nacional de Incentivos en la Secretaría de Investigación y post grado de la Universidad Nacional de Misiones, Argentina

Temáticas de interés: inclusión educativa de personas con discapacidad, educación inclusiva

\section{City I Ciudad:}

La Plata [ar]

e-mail:

posgrado@fahce.unlp.edu.ar

Citar como:

Schewe, C. L. (2017). Autoridad, reconocimiento y emancipación: Educación de personas en situación de discapacidad. Horizontes Pedagógicos, issn-l:0123-8264, 19 (2), 69-76. Obtenido de: https://revistas.iberoamericana.edu.co/index. php/rhpedagogicos/article/view/1157 


\section{Autoridad, reconocimiento y emancipación Educación de personas en situación de discapacidad}

Authority, recognition and emancipation in the education of people with disabilities

Carolina Lelia Schewe

\section{Trayectorias educativas}

La educación inclusiva es una de las alternativas más urgentes para el ejercicio del derecho a la educación de todas las personas, independientemente de sus condiciones de vida. El concepto de inclusión, "guarda relación directa con lo sucedido con su opuesto, el concepto de exclusión: ambos conceptos comenzaron a ser usados crecientemente para poner en palabras las principales líneas que trazan el dibujo de la sociedad contemporánea”. (Pérez \& Krichesky, 2015, pág. 29)

Con respecto a las personas con discapacidad, el desafío recurrente se presenta con la necesidad de conformar espacios inclusivos de aprendizaje (García García \& Cotrina García, 2017; Moliner García, Sales Ciges, \& Escobedo Peiro, 2016; Blanco Guijarro, 2006), en todos los niveles y modalidades del sistema educativo, con respecto a la currícula, la cultura y las comunidades, para que no existan situaciones de exclusión por las barreras sociales existentes en las instituciones. La discapacidad -entonces- se representa "como una situación que atraviesa una persona, que puede ser permanente o transitoria, y que de ningún modo define su capacidad de ser en el mundo”. (Barrozo, 2015, pág. 3).

Este trabajo presenta una serie de reflexiones, referidas a la configuración de la autoridad en las trayectorias educativas escolares de personas con discapacidad, en dos diferentes y posibles escenarios: las escuelas de Educación Especial, como espacio institucional al que deben concurrir las personas con discapacidad exclusivamente y las escuelas de educación común, como espacios a los que recurren todos los estudiantes.

La legitimidad de la palabra de los profesionales -que deciden a cuál de los dos espacios deben concurrir los estudiantes- está dada por la historia de las conceptualizaciones que han sostenido a la Educación Especial, constituyendo el discurso hegemónico (Skliar \& Pérez, 2013; Pérez, 2014), o dominante (Zardel Jacobo, 2016), hasta nuestros días. Sostenemos con Katz (2016), que "si bien en los últimos años se viene dando una mayor presencia de discursos y prácticas críticas [...], el conocimiento producido en discapacidad ha estado históricamente dominado por los discursos biologicistas, esencialmente el discurso médico y psicopedagógico". (pág. 17). La relación que existe entre esos discursos y la construcción de los procesos que mencionamos, está estrechamente ligada a "la herencia del deber ser" (Pérez \& Gallardo, 


\section{Autoridad, reconocimiento y emancipación}

Educación de personas en situación de discapacidad

Derecho, 2016; Vain, 2015), que no contempla lo que en cada caso particular se inaugura en la relación con un Otro. Ocampo (2016, pág. 22), afirma que el discurso de la educación inclusiva es -justamente- una oportunidad para reexaminar estas prácticas y las producciones al respecto porque:

\begin{abstract}
... otorga elementos para cartografiar la identidad y naturaleza científica de este modelo, con el propósito de cuestionar su campo conceptual, ideológico, político, ético y pedagógico. Abre, además, la posibilidad de enriquecery modernizar las bases teóricas e investigativas vinculadas a la Educación Especial, por tanto, es una reformulación de la sociedad, de la política y de la educación en general.

(Ocampo González, 2016, pág. 22)
\end{abstract}

Afirmamos, siguiendo a los autores, que la educación inclusiva debe ser concebida como la construcción de un proyecto político, histórico y ético más amplio (Ainscow, 2001; Ocampo González, 2016; Pérez \& Gallardo, 2016), lo que posibilitaría una reconfiguración de la autoridad en vistas a proporcionar espacios de participación y toma de decisiones de las personas con discapacidad, sobre sus propias trayectorias.

\section{Metodología}

El presente trabajo se origina a partir de una revisión teórica que trata de identificar los aspectos centrales de la autoridad pedagógica, en los procesos educativos formales de personas con discapacidad.

Arendt (1972) afirma que, en la historia del pensamiento político, la autoridad como imperativo natural sirvió para sostener formas autoritarias de gobierno, para regir relaciones entre adultos y niños, entre profesores y alumnos, para brindar estabilidad. Esto significa que, para el análisis que proponemos, es necesario retomar las construcciones históricas en el campo de la Educación Especial, que legitiman y circunscriben las decisiones sobre las trayectorias educativas de las personas con discapacidad (Schewe, 2017), teniendo en cuenta que, actualmente, el Modelo Social de la discapacidad (Palacios Rizzo \& Bariffi, 2014), propone la eliminación de las barreras que se encuentran en los entornos de desarrollo de las personas (y no en las personas, sus discapacidades, sus limitaciones). Ahora bien, a la hora de implementar los procesos propuestos desde las políticas de inclusión educativa de personas con discapacidad, ¿cuáles son las marcas de desigualdad que determinan la posición de los estudiantes? (Ocampo González, 2012, pág. 135), ¿quiénes toman las decisiones?, ¿cómo participan las personas con discapacidad?

Las preguntas que guían las reflexiones tienen que ver con categorías teóricas tomadas de Greco (2007), quien -desde la Filosofía de la Educación- plantea que el análisis:

... proviene fundamentalmente del territorio de la filosofía - pero también de la psicología, la política, la pedagogía y el psicoanálisis-como plataformas de partida y cruce de caminos, no como espacios disciplinares cerrados sobre sí. Territorios sin fronteras fijas, tal vez mapas superpuestos que entrelazan los caminos, itinerarios geográficos, pero también su relato, allí donde se cuenta lo que el mapa no alcanza a mostrar. Desde este recorrido fue posible desplegar pensamientos y acciones entre disciplinas o porfuera de ellas, de bordes móviles y habilitadores de inesperados trayectos. 'Rodeos teóricos' que no dejan de dar vueltas en torno a su objeto en forma inconclusa y que recomienza cada vez.

(Greco, 2007, pág. 7)

Considerando que, a partir de las normativas actuales sobre la educación de personas con discapacidad (Arango Mira \& Yarza de los Ríos, 2013), deberían construirse procesos que habiliten la participación de todas las personas en todos los espacios educativos (de la Rosa Moreno, 2013), otra pregunta que funciona como punto de partida para estas reflexiones es ¿cómo se configura la autoridad pedagógica en los procesos de inclusión de personas con discapacidad a instituciones de educación común?

También, retomamos los planteos de Greco (2007) respecto a ¿Qué puede una autoridad en el territorio de la educación? formulándola, en este caso con respecto al campo de la Educación Especial; entonces ¿Qué puede una autoridad en la Educación Especial?

\section{Resultados}

En referencia al concepto de autoridad (Greco, 2016), en este trabajo presentamos la postura de Arendt (Arendt, 1972), quien afirma que la autoridad es la capacidad de introducir al otro en una historia construyendo una trama de sentidos. Como se ha afirmado al inicio, desde el año 2006, en el marco de la Ley de Educación Nacional 26.206, el sistema educativo en Argentina, se ha redefinido. Para las escuelas de Educación Especial, se propuso que en lugar de recibir a aquellos estudiantes "imposibilitados", puedan convertirse en espacios de apoyo, para garantizar trayectorias educativas integrales de los/as alumnos/as con discapacidad" en los espacios educativos que ellos elijan. Se considera a la inclusión educativa como un concepto posible, para el que:

\section{... se debe tener en cuenta la palabra del sujeto-alumno al momento de la toma de decisiones [... y se ha instalado la necesidad de desarrollar intervenciones pedagógicas que vayan de la individualización de la acción pedagógica hacia la idea de una individualización de los itinerarios formativos.}

(Ministerio de Educación, Ciencia y Tecnología, 2009)

Las cuestiones como los recursos, los apoyos (Carvajal Osorio \& Cruz Perdomo, 2014), la formación docente, las dificultades en la implementación de políticas y la falta de normativas jurisdiccionales, dieron como resultado que las escuelas de Educación Especial -actualmente- reciban en sus aulas a estudiantes con necesidades educativas especiales por problemas de conducta, falta de atención, condiciones sociales desfavorables, entre otros factores. Esto implica que, al culminar sus estudios, los estudiantes no acceden a la acreditación del nivel primario, lo que les impide continuar con su escolarización formal en el nivel secundario (y superior). La única alternativa en estos casos, consiste en recurrir a otras modalidades o estrategias de acreditación, como programas "especiales" o escuelas de reingreso. Por esto, planteamos en este trabajo que "hablar de autoridad es hablar de vínculos, de estar juntos, de una trama de encuentros" (Greco, 2007), que necesariamente, en este caso refieren al ejercicio de cada uno de los elementos de la autoridad (ídem):

\section{Recepción de nuevas generaciones}

La responsabilidad sobre la atención temprana y la iniciación de procesos educativos, son algunas de las cuestiones que atraviesan estos espacios. Los profesionales que trabajan en las escuelas de Educación Especial son quienes asesoran a padres y docentes de educación común sobre las opciones para la educación de sus hijos. La recepción de los niños con discapacidad en espacios que les proporcionen herramientas para crecer, tiene que ver con "sostener 
la idea de que a los adultos en las escuelas nos cabe la función, la responsabilidad de preservar al niño ejerciendo, ejercitando nuestro papel de mediadores con la realidad, porque esa mediación opera como pantalla protectora". (Zelmanovich, 2003, pág. 4)

\section{Nombrar y Reconocer}

La enseñanza en las escuelas de Educación Especial se encuentra fuertemente ligada a la subjetividad en vías de constitución (Zelmanovich, 2003), de los estudiantes. El educador conoce la necesidad de los niños o adolescentes de inscribirse en una trama de sentidos, para comprender cómo se construyen sus representaciones sobre el mundo. Nombrar y reconocer, como parte de este proceso implica la escucha por parte de los adultos, quienes intervienen explicando, haciendo parte al estudiante de las escenas y reconociendo su posibilidad de comprender e intervenir en futuras situaciones, fuera de esos espacios.

\section{Proteger la vida}

Algunas personas con discapacidad están sometidas a varias formas de exclusiones y otras formas de violencias (De la Vega, 2015; Venturiello, 2013). En estos casos, la reconfiguración de la autoridad implica retomar el lugar -de niño o adolescente- del estudiante, sin desestimar su posibilidad de comprender la situación. Zelmanovich (2003), afirma que:

... la posibilidad de dar sentido a lo que se ubica en los confines de la racionalidad, se hace factible si hay un Otro que mantiene algún grado de integridad para situar en una trama lo que irrumpe de la realidad.

(Zelmanovich, 2003)

En este caso, el Otro, es el sostén que el estudiante necesita para poder acceder a la cultura. Otros autores confirman los peligros de que se generen "procesos que están siendo cada vez más naturalizados en la cotidianeidad escolar y que implican circuitos escolares diferenciados para estos niños/as que terminan siendo derivados a una oferta educativa 'diferente' como lo es la educación especial” (Pereyra, 2015), por las implicancias para su subjetividad.

\section{Autorizar lo nuevo}

El reconocimiento de las personas con discapacidad como sujetos de derecho a la educación en todos los niveles y modalidades del sistema educativo constituye "lo nuevo", dada la historia de imposibilidades atribuidas a las personas con discapacidad. Esta comprensión implica, lo que varias escuelas de Educación Especial asumieron como parte de su trabajo (Schewe, 2014): difundir la necesidad de la creación de culturas inclusivas para personas con discapacidad en todos los espacios posibles.

En las escuelas de educación común, son las escuelas de Educación Especial (o los especialistas en el campo) las que "autorizan" y en muchos casos habilitan, los procesos de trabajo con personas con discapacidad. Se constituyen como quienes legitiman las posibilidades de los estudiantes.

\section{Trazar líneas de continuidad entre generaciones}

La autoridad como sostén retoma cada acto como una oportunidad de emancipación (Greco, 2007; Milano Fulchi, 2015; Aleu, 2008). En el orden de la aparición de lo nuevo (que mencionábamos en el punto e), se interrumpen las dinámicas sociales con respecto a la derivación de las personas con discapacidad a espacios que históricamente estaban destinados exclusivamente a su atención. Esto implica, para algunos autores como Slee (2011) y Thomas y Loxley (2007), la necesidad de una deconstrucción de la Educación Especial y la construcción de la Educación Inclusiva como una alternativa, insistiendo en que la solidez de los conocimientos propios de este campo "racional y sensible” (2007, pág. 13) constituyen un impedimento.

Esto significa que, existe una responsabilidad de autoridad, de decidir cuáles son los elementos que continuarían con las configuraciones de apoyo necesarias para que la sociedad asuma "lo nuevo". Retomamos aquí la idea de reconfiguración, dado que es imposible seguir sosteniendo los planteos tradicionales del campo -desde su sesgo paternalista- (Kojéve, 2004), -médico y asistencialista(Skliar, 1997; Slee, 2011), y asumir - a su vez- la necesaria articulación con los demás niveles y modalidades del sistema educativo para el desarrollo pleno de los estudiantes.

\section{Interrumpir los órdenes injustos}

Lo dicho hasta aquí, nos anticipa sobre lo que Zelmanovich (2003), llama "debilitamiento del tejido simbólico que estructura los ideales y las creencias", que la escuela retoma y "atempera, que protege, que resguarda, y que posibilita por esa vía el acceso a la cultura" (2003). Los órdenes injustos contra las personas con discapacidad llevan siglos de construcción histórica y es la escuela uno de los espacios principales para su interrupción, a partir de la educación inclusiva.

Esos significados [...] le permiten al alumno construir su diferencia, que es su propia palabra y en ello va la asimetría, la protección y el reconocimiento de la vulnerabilidad del niño. De allí la necesidad de pensar y operar sobre las dificultades que tenemos hoy los adultos para sostener una asimetría frente a los chicos, que constituye, en definitiva, el soporte de esa trama de significados que ampara y protege.

(Zelmanovich, 2003, pág. 29)

\section{En el terreno de lo singular y lo político}

La necesidad de una praxis pedagógica, emancipatoria, igualitaria, en el campo de la Educación Especial radica en la urgencia de que los estudiantes puedan organizarse para ejercer sus derechos. Uno de los objetivos que perseguimos quienes trabajamos en vistas a generar espacios inclusivos de aprendizaje es que los estudiantes puedan convertirse en ciudadanos capaces de tomar decisiones sobre sus vidas, en palabras de Rancière (2003), "no como alumnos [.. . ] sino como hombres [y mujeres], como se responde a alguien que os habla y no a alguien que os examina: bajo el signo de la igualdad", confiando en la capacidad intelectual de todo ser humano.

La intervención de los estudiantes en el ámbito de lo político, debe ser planificada teniendo en cuenta que:

... lo propio de nuestra época es que las estrategias que permitían hacer frente a la desprotección, han dejado de ser colectivas. Las instituciones que ofrecían protección, hoy sufren un paulatino debilitamiento, provocando una sensación de indefensión sin precedente. Al mismo tiempo, el desgarramiento del lazo social deja al individuo aislado en el miedo.

(Cantarelli, 2006, pág. 4)

Las posibilidades de afrontar este desafío se visualizan y se vivencian solamente si existen esas palabras que transportan (Greco, 


\section{Discusión}

Las posibilidades de aprendizaje en los espacios escolares, son parte de las trayectorias (Nicastro \& Greco, 2012; Pérez, Gallardo, \& Schewe, 2017), a las que acceden -y que pueden construir- los estudiantes con discapacidad. Actualmente,

\begin{abstract}
se han visibilizado grupos que históricamente se han encontrado en riesgo de no contar con las condiciones para acceder, mantenerse y egresar de la escuela, hecho que vulnera su dignidad humana y sus derechos fundamentales como ciudadano. En el caso de la población declarada con alguna condición de discapacidad, la instalación de dichos lineamientos de política de educación inclusiva, ha sido valioso en el proceso que se vive en el país, para contrarrestar las dinámicas de desigualdad presentes en la escuela que ponen en desventaja a algunos o muchos escolares al no ser considerados aptos para aprender. (Carvajal Osorio \& Cruz Perdomo, 2014, pág. 108)
\end{abstract}

La imposibilidad de acceder a los espacios educativos comunes es, en primera instancia, una decisión de profesionales docentes, psicopedagogos, psicólogos, médicos, que consideran que la discapacidad es una condición para decidir (a priori) sobre los destinos educativos de los estudiantes. Ordoñez Mora, Díaz Elejalde, Lozada Reyna, Flórez Valencia y Gómez Ramírez (2016), afirman que:

\section{... en la actualidad, se han evidenciado algunas} dificultades para la población con discapacidad en cuanto a acceso equitativo a los servicios de salud, educación y oportunidades laborales se refiere, pues muchas veces no reciben los servicios que necesitan de acuerdo a la discapacidad que presentan, situación que en ocasiones limita el desarrollo de actividades de la vida cotidiana y restringe su participación en algunos espacios.

(2016, pág. 127)

Estas decisiones atraviesan la infancia, que representa -en este caso- una doble imposibilidad: la de elegir a qué espacios educativos se concurre y la de participar de ciertos espacios destinados a la infancia por la condición de discapacidad. La infancia, en palabras de Penchaszadeh y Biset (2013) representa -no solamente- el momento negado e inmemorial del humano, sino todos los "no dichos" que habitan espectralmente nuestras sociedades. La autora afirma que las instituciones que deben velar por el bienestar de las personas terminan cometiendo aberraciones, porque la hospitalidad sin "la conciencia de la hostilidad que ella misma entraña", solo puede culminar con la aparición "amorfa” de lo reprimido (Penchaszadeh \& Biset, 2013, pág. 11).

La autoridad, en este caso, sostiene y delimita determinados lugares (Greco, 2007), excluyendo a los estudiantes por su condición, apoyándose en excusas. En palabras de Skliar,

... la respuesta de parte de la escuela común casi siempre es: "no estamos preparados", o bien: "no estoy preparado para atender a ese niño, a esa niña, a ese joven". (...) A esta altura de los acontecimientos es posible afirmar que no sabemos qué significa estar preparados. ¿Qué puede significar la expresión "estar preparados" o "no estar preparados"? ¿Qué puede entenderse de esa afirmación a propósito de un supuesto "saber qué hacer" ante cada niño, ante cada cuerpo, ante cada lengua, ante cada aprendizaje, ante cada forma de estar en el mundo? [...] Es un imposible saber, sentir y estar preparado para aquello que pudiera venir. Hay que enfatizar la idea de que más que estar preparados, anticipados a lo que vendrá, que nunca sabemos que es, de lo que se trata es de estar disponibles y de ser responsables.

(Skliar, 2008, pág. 10)

El autor sostiene que la disponibilidad y la responsabilidad son cuestiones correspondientes a la ética, que la hospitalidad que requiere el ser docente significa estar disponible para recibir a quien sea, a cualquiera, a todos, a cada uno. Por otro lado, retomamos aquí lo planteado por Greco (2007), en cuanto a que la sociedad ha transformado a niños y jóvenes en sus enemigos, inhabilitando sus capacidades, perdiendo su derecho a la educación y a elegir su proyecto devida, no se advierte un hacerse cargo de los niños y jóvenes como responsabilidad de los adultos. Una de las investigaciones que consideramos relevante con respecto a este tema corresponde al trabajo de Calderón Almendros y Habegger Lardoeyt (2012), en el que se plantea la lucha de una familia de una persona con Síndrome de Down, contra la escuela, para demostrar que su hijo/hermano podía cursar toda su trayectoria escolar en la escuela regular hasta llegar a la universidad y obtener el grado superior de música. Esto implica romper con el orden natural "de quienes nacieron para pensar, actuar socialmente, saber y poder sobre los que nacieron para las manualidades y cuya palabra no tiene lugar, no cuenta". (Greco, 2007). Al mismo tiempo, se presenta como obstáculo,

... la rigidez de las instituciones formales para estructurar el conocimiento (en niveles desconexos) y la actividad académica (por cursos, trimestres, incluso evaluaciones), la delimitación de los espacios (por clases cerradas sin un proyecto común) y de los tiempos (una hora por materia, cada una sin permear las demás), la diferenciación de un profesorado independiente y disgregado (por disciplinas y áreas de conocimiento, así como por niveles) y el énfasis en una disciplina que atenta contra la curiosidad misma de los chicos y chicas por el aprendizaje (el permanecer sentados, el hacer lo que dice el profesorado...), son algunas de las razones más chocantes de la organización de la actividad en las instituciones educativas formales. Esta obsesión por clasificar, delimitar y estructurar acaba, casi obligadamente, por situar a las personas con hándicap en el punto de mira al no encontrarse dentro de los patrones de la normalidad.

(Calderón Almendros \& Habegger Lardoeyt, 2012, pág. 150)

La responsabilidad de habilitar palabras que autorizan (2012), es parte del trabajo en las instituciones educativas, particularmente, en el caso de las que se constituyen como espacios inclusivos de aprendizaje para personas con discapacidad: es necesario que los adultos sostengan la confianza (Cornú, 2004), para poder ejercer la mediación que supone que los otros (en este caso, estudiantes) puedan renunciar a reaccionar en contra de las acciones que supone la autoridad, en el sentido que le atribuye Kojéve. Esta actitud implica como eje central del trabajo, la participación (Schewe, 2011) y "pensar de manera compleja y complejizada” (Katz, 2013), los sentidos más profundos de la educación.

Susinos Rada y Latas Parrilla (2013), proponen como alternativa, un "camino de la construcción de un conocimiento que sea inclusivo" (pág. 89), priorizando la voz protagónica de los procesos, a aquellos que resultan excluidos. Este camino resulta necesario, teniendo en cuenta que "no se han podido transformar las limitaciones de la sociedad, para prestar servicios apropiados y para asegurar adecuadamente que las necesidades de todas las personas sean tenidas en cuenta dentro de la organización social”. (Sosa, Acuña, Conese, \& Mirc, 2015, pág. 14) 


\section{Conclusiones}

El desafío que se plantea para los procesos de educación inclusiva nos invita a "hablardeemancipación situada en nuestra épocayen nuestras condiciones para promover el despliegue de las posibilidades de cada uno: que haga oír su voz, que encuentre su propia escritura". (Greco, 2007). Siguiendo a la autora, retomamos que resulta difícil historiar el propio tiempo y hacernos cargo de la exigencia de pensar qué hacer en el medio de tiempos de transformación, aunque es ineludible hacerlo: en épocas pasadas existían jerarquías institucionales cuya obediencia marcaba los rumbos de las vidas de las personas, actualmente, en el marco de la horizontalidad de las instituciones se hace evidente la necesidad de preguntarnos sobre los lugares que circunscriben las prácticas institucionales.

En este sentido, López Melero (2012, pág. 136), advierte una contradicción basada en que "a menudoexigimos a losjóvenes quesean personas solidarias y cooperativas y, sin embargo, los empujamos a diario a la competitividad, cuando la competitividad es, precisamente, la negación de la cooperación". La autoridad emancipadora (Arendt, 1972; Greco, 2007; Alegre, y otros, 2012) posibilita; la autoridad debilitada, sostiene las imposibilidades. El maestro (como adulto, profesional de la educación y parte fundamental de los procesos de inclusión de los estudiantes) debe estar emancipado, en primer lugar, porque su trabajo es político y en segundo lugar porque de su intervención dependen, en gran medida, las posibilidades futuras de los estudiantes. En palabras de Ranciére (2003): "aprenderá [...] si el maestro cree que puede y si le obliga a actualizar su capacidad".

A partir de estas reflexiones, vemos cómo se configura la autoridad con respecto a laeducación inclusiva: en un doblejuego de posibilidades e imposibilidades. Este trabajo se denominó originalmente autoridad es la que te enseña sin faltarte el respeto, a partir de la expresión de un adolescente -que participó en una instancia de formación en una escuela de Educación Especial- que remite a una de las escenas más recurrentes en la educación de personas con discapacidad: la desconfianza en los procesos del estudiante, la falta de respeto más incapacitante.

Volviendo, entonces, a la pregunta que planteamos al inicio de este trabajo ¿qué puede una autoridad en la Educación Especial? y a partir de los elementos que hemos considerado para la construcción de este trabajo, podemos afirmar que, una autoridad en el campo de la Educación Especial puede determinar -en gran medida- el futuro y las oportunidades de vida de las personas con discapacidad.

Este artículo es una invitación a tener en cuenta las implicancias de las decisiones, que durante mucho tiempo estuvieron -e inclusive sigue estando hoy- libradas a la aplicación de un test, a un estado de salud o a un prejuicio.

\section{Referencias}

Ainscow, M. (2001). Desarrollo de Escuelas Inclusivas: Ideas, propuestas y experiencias para mejorar las instituciones escolares (308 ed., Vol. 84 Educación Hoy Estudios). Madrid [es]: Narcea.

Alegre, S., Fernández Tobal, C., Benedetti, E., Levaggi, G., Lisnevsky, A., \& Villegas, A. M. (2012). Nuevas Configuraciones de lo Escolar: Pensar la autoridad pedagógica emancipatoria en la educación de jóvenes y adultos. IV Congreso Internacional de Investigación y Práctica Profesional en Psicología XIX Jornadas de Investigación VIII Encuentro de Investigadores en Psicología del MERCOSUR. XXI, págs. 305-310. Buenos Aires [ar]: Universidad de Buenos Aires. Obtenido de http://www. scielo. org.ar/pdf/anuinv/v19n1/v19n1a32.pdf

Aleu, M. (2008). Las Concepciones de Autoridad en los Estudiantes de la Escuela Media [reseña]. Propuesta Educativa(31), 129-131. Obtenido de http:// www. propuestaeducativa. flacso.org. ar/tesis.php? num=31\&id=17
Arango Mira, P. A., \& Yarza de los Ríos, A. (2013). ¿Aprender Juntos o Aprender Separados?: Relatos de vida sobre las experiencias de aprender de niños y niñas con discapacidad (es) en Medellín (Antioquia, Colombia) Horizontes Pedagógicos, 15(1), 69-82. Obtenido de http://revistas. iberoamericana.edu.co/index.php/rhpedagogicos/article/view/406

Arenas, F. D., \& Sandoval Saenz, M. (2013). Procesos de Flexibilización y Diversificación Curricular: Nuevos retos del sistema educativo colombiano para favorecer los procesos de participación en contextos escolares de personas con discapacidad. Horizontes Pedagógicos, 15(1), 147-157. Obtenido de http://revistas.iberoamericana.edu.co/index.php/ rhpedagogicos/article/view/42I

Arendt, H. (1972). La Crisis de la Cultura. En H. Arendt, Entre el pasado y el futuro: Ocho ejercicios sobre la reflexión política [Between Past and Future] (A. Poljak, Trad., págs. 269-302). París [fr]: Peninsula; Penguin; Gallimard.

Barrozo, N. N. (2015). Análisis Aproximativo sobre la Extensión de la Obligatoriedad de la Escuela Secundaria para Personas con Discapacidad en la Provincia de Salta. XII Jornadas Argentinas de Estudios de Población (pág. 14). Salta [ar]: Universidad Nacional de Salta. Obtenido de http:// www.economicas.unsa.edu.ar/web/archivo/otros/AEPA/SESION-3/Sesion3-Barrozo.pdf

Blanco Guijarro, R. (2006). La Equidad y la Inclusión Social: Uno de los desafíos de la educación y la escuela hoy. Reice: Revista Iberoamericana sobre Calidad Eficacia y Cambio en Educación, 4(3), 1-15. Obtenido de http:// www.rinace.net/arts/vol4num3/art1.htm

Calderón Almendros, I. (2013). Entre la Realidad y los Sueños: Reflexiones sobre la inclusión educativa desde la vida de mi hermano. Revista de Educación Inclusiva, 6(1), 156-159. Obtenido de http://www. revistaeducacioninclusiva.es/index.php/REl/article/view/200

Calderón Almendros, I., \& Habegger Lardoeyt, S. (2012). Educación, Hándicap e Inclusión: Una lucha familiar contra una escuela excluyente (Vol. Recursos Octaedro Andalucía). Granada [es]: Mágina.

Cantarelli, M. (2006). Después de la familia tipo, ¿qué? A propósito de Historias de familia. Dirección Nacional de Gestión Curriculary Gestión Docente, Ministerio de Educación, Ciencia y Tecnología de la Nación. Ciudad de Formosa [ar]: Ministerio de Educación, Ciencia y Tecnología de la Nación. Obtenido de http://www.me.gov.ar/curriform/publica/cantarelli familiatipo.pdf

Carvajal Osorio, M. M., \& Cruz Perdomo, J. (2014). Los Apoyos para la Participación del Escolar con Discapacidad. Horizontes Pedagógicos, 16(1), 106 119. Obtenido de http://revistas.iberoamericana.edu.co/index.php/ rhpedagogicos/article/view/782

Cornú, L. (2004). Transmisión e Institución del Sujeto: Transmisión simbólica, sucesión, finitud. En La transmisión en las sociedades, las instituciones y los sujetos: Un concepto de la educación en acción (pág. 232). Buenos Aires [ar]: CEM-Novedades Educativas.

de la Rosa Moreno, L. (2013). Detalles de la Historia de Vida de José Ángel Lobato Carvajal: "Buscando la normalidad en un mundo anormal". Revista de Educación Inclusiva, 6(1), 107-128. Obtenido de http://www. revistaeducacioninclusiva.es/index.php/REl/article/view/197

De la Vega, E. (2015). Lógica de las Nuevas Violencias: Ilusiones sobre el porvenir de la escuela. Buenos Aires [ar]: Noveduc.

García García, M., \& Cotrina García, M. J. (2017). La Contribución de la Universidad al Desarrollo de Prácticas Inclusivas: Dilemas y propuestas para avanzar compartiendo. Revista de Educación Inclusiva, 5(1), 123-138. Obtenido de http://www.revistaeducacioninclusiva.es/index.php/REl/article/view/226

Greco, M. B. (2007). La Autoridad (Pedagógica) en Cuestión (Vol. Enfoques y perspectivas). Buenos Aires [ar]: Homo Sapiens.

Greco, M. B. (2016). Democracia y Políticas Emancipatorias: La pregunta filosófico-política de la autoridad en el ámbito de la formación docente universitaria. Revista Electrónica Instituto de Investigaciones Jurídicas y Sociales A. L. Gioja, 14, 104-124. Obtenido de http://www.derecho.uba.ar/ revistas-digitales/index.php/revista-electronica-gioja/article/view/28

Katz, S. L. (2013). La Investigación en Relación a la Discapacidad en la Educación Superior: Un acercamiento latinoamericano. IV Congreso Internacional de Investigación de la Facultad de Psicología de la Universidad Nacional de La Plata (págs. 354-355). La Plata [ar]: Universidad Nacional de La Plata. Obtenido de http://sedici.unlp.edu.ar/handle/10915/48687

Katz, S. L. (2016). El Sentido de una Red Universitaria Latinoamericana sobre Discapacidad y Derechos Humanos. Diálogos e Perspectivas em Educação Especial, 2(2), 9-22. Obtenido de http://www2.marilia.unesp.br/ ojs-2.4.5/index.php/dialogoseperspectivas/article/view/5753

Kojéve, A. (1942). La Noción de Autoridad (125 ed.). Buenos Aires [ar]: Nueva Visión. 


\section{Autoridad, reconocimiento y emancipación}

Educación de personas en situación de discapacidad

Ley 26.206, Ley Nacional de Educación (Congreso de la Nación Argentina 27 de 12 de 2006). Obtenido de http://servicios.infoleg.gob.ar/infoleglnternet/ anexos/120000-124999/123542/norma.htm

López Melero, M. (2012). La Escuela Inclusiva: Una oportunidad para humanizarnos. Revista Interuniversitaria de Formación del Profesorado(74), 131-160. Obtenido de http://www.aufop.com/aufop/ uploaded files/articulos/1396551140.pdf

Milano Fulchi, T. (2015). Prácticas de Cuidado Infantil y Sensibilidad Paterna en Relación a las Representaciones Sociales de Paternidad Subyacentes. Montevideo [uy]: Universidad de la República.

Ministerio de Educación, Ciencia y Tecnología. (2009). Recomendaciones para la Elaboración de Diseños Curriculares: Profesorado de educación especial. Buenos Aires [ar]: Ministerio de Educación, Ciencia y Tecnología [ar] Instituto Nacional de Formación Docente. Obtenido de http://www.feeye. uncu.edu.ar/web/XVII-JN-RUEDES/Educacion Especial INFOD.pdf

Moliner García, O., Sales Ciges, A., \& Escobedo Peiro, P. (2016). Posibilidades y Limitaciones de Generar Cultura de Centro desde las Prácticas Compartidas de Aula: El caso de la educación intercultural inclusiva. Estudios sobre Educación, 30, 51-70. doi:10.15581/004.30.51-70

Nicastro, S., \& Greco, M. B. (2012). Entre Trayectorias: Escenas y pensamientos en espacios de formación (156 ed., Vol. Enfoques y perspectivas). Santa Fe [ar]: Homo Sapiens.

Ocampo González, A. A. (2012). Mejorar la Escuela Inclusiva: Un desafío de todos. Revista de Educación Inclusiva, 5(3), 133-141. Obtenido de http://www. revistaeducacioninclusiva.es/index.php/REl/article/view/248

Ocampo González, A. A. (2016). Aproximaciones y Descripciones Generales sobre la Descripción del Objeto de la Educación Inclusiva. En Los Rumbos de la Educación Inclusiva en Latinoamérica en los inicios del Siglo XXI: Cartografías para modernizar el enfoque (Vol. 1 Cuadernos de Educación Inclusiva, págs. 24-89). Santiago [cl]: Celei, Centro de Estudios Latinoamericanos de Educación Inclusiva. Obtenido de https://dialnet. unirioja.es/descarga/articulo/5578424.pdf

Ordoñez Mora, L. T., Díaz Elejalde, L. P., Lozada Reyna, V., Florez Valencia, L. M., \& Gómez Ramírez, E. (2016). Procesos de Inclusión Escolar Mediados por Fisioterapia en Niños de 0 a 16 Años. Horizontes Pedagógicos, 18(1), 126-136. Obtenido de http://revistas.iberoamericana.edu.co/index.php/ rhpedagogicos/article/view/907

Palacios Rizzo, A., \& Bariffi, F. J. (2014). La Discapacidad como una Cuestión de Derechos Humanos: Una aproximación a la convención internacional sobre los derechos de las personas con discapacidad (144 ed., Vol. 4 Colección Telefónica Accesible). (E. Trujillo Jiménez, \& L. C. Perez Bueno, Edits.) Madrid [es]: Telefónica; Cermi; Ediciones Cinca. Obtenido de http:// www.repositoriocdpd.net:8080/bitstream/handle/123456789/402/L PalaciosA_DiscapacidadDerechosHumanos_2007.pdf?sequence=1

Penchaszadeh, A. P., \& Biset, E. (2013). Derrida Político. Buenos Aires [ar]: Colihue.

Pereyra, C. (2015). La Configuración de Circuitos Diferenciados Derivados a Educación Especial en Contextos de Pobreza. Ruedes, 4(6), 58-73. Obtenido de http://bdigital.uncu.edu.ar/objetos digitales/6906/04 pereyra-ruedes4-6.pdf

Pérez, A. V. (2014). Educación Especial y Alteridad: En busca de lo común. Revista latinoamericana de educación inclusiva, 8(1), 153-169. Obtenido de http://www.rinace.net/rlei/numeros/vol8-num1/art10.html

Pérez, A. V., \& Gallardo, H. H. (2016). Derecho: Derechos y (dis)capacidad. Pasajes(2), 32-47. Obtenido de http://wwW.revistapasajes.com/ gallery/3\%20 ficial\%20articulo\%202016\%20dra.\%20andrea\%20\%20 veronica\%20perez\%20y\%20lic.\%20hector\%20hugo\%20gallardo.pdf

Pérez, A. V., \& Krichesky, M. (2015). La Escuela Secundaria en el Foco de la Inclusión Educativa. Buenos Aires [ar]: Universidad Nacional de Avellaneda.

Pérez, A. V., Gallardo, H. H., \& Schewe, C. L. (2017). Acerca de los Orígenes Históricos de las Desigualdades Educativas en la Argentina: Discapacidad y derecho a la educación hoy. En A. A. Ocampo González, Formación de maestros e investigadores para la educación inclusiva: perspectivas críticas. Santiago [cl]: Celei, Centro de Estudios Latinoamericanos de Educación Inclusiva.

Rancière, J. (2003). El Maestro Ignorante: Cinco lecciones sobre la emancipación intelectual (184 ed., Vol. 103 Laertes Educación). (N. Estrach Mira, Trad.) Barcelona [es]: Sedición.
Schewe, C. L. (2011). La Educación Especial desde una Perspectiva Sociocomunitaria: Una experiencia sobre la relocalización en la Escuela Especial N45. Ruedes, 1(2), 133-138. Obtenido de http://bdigital.uncu. edu.ar/3948

Schewe, C. L. (2014). Las Escuelas de Educación Especial en Misiones: Metas y objetivos. I Simposio del Observatorio de la Discapacidad (págs. 422 440 [653]). Bernal [ar]: Universidad Nacional de Quilmes. Obtenido de http://observatoriodiscapacidad.web.unq.edu.ar/wp-content/uploads/ sites/32/2014/09/1-Simposio-Internacional-del-Observatorio-de-laDiscapacidad-2014-isbn.pdf

Schewe, C. L. (2016). Políticas de Inclusión Educativa de Personas con Discapacidad: Continuidades y discontinuidades en los procesos para su implementación. Actas del II Simposio del Observatorio de la Discapacidad - Políticas públicas, ideologías y modos de abordaje de la discapacidad en el marco de las Ciencias Sociales (págs. 260275 [870]). Bernal [ar]: Universidad Nacional de Quilmes. Obtenido de http://observatoriodiscapacidad.web.unq.edu.ar/wp-content/ uploads/sites/32/2014/09/Actas-del-II-Simposio-del-Observatorio-de-LaDiscapacidad.pdf

Schewe, C. L. (2017). Discapacidad, Educación Especial y Educación Inclusiva. Revista Internacional de Educación y Aprendizaje, 5(1). Obtenido de http://journals.epistemopolis.org/index.php/educacion/article/view/1465

Skliar, C. (1997). La Educación de los Sordos: Una reconstrucción histórica, cognitiva y pedagógica (187 ed.). Mendoza [ar]: Universidad Nacional de Cuyo.

Skliar, C. (1997). Una Mirada sobre los Nuevos Movimientos Pedagógicos en la Educación de los Sordos. XX Reuniao Anual do ANPED, Associacao Nacional de Pesquisadores em Educacao. Mendoza [ar]: Anped, Associacao Nacional de Pesquisadores em Educacao. Obtenido de http://www.cultura-sorda.org/wp-content/uploads/2015/03/Una-miradasobre-los-nuevos-movimientos-pedagogicos.pdf

Skliar, C. (2008). ¿Incluir las diferencias? Sobre un problema mal planteado y una realidad insoportable. Orientación y Sociedad, 8, 1-17. Obtenido de http:// wWw. scielo.org.ar/pdf/orisoc/v8/v8a02.pdf

Skliar, C., \& Pérez, A. V. (2013). Lo Jurídico y lo Ético en el Campo de la Educación: La enunciación de la diversidad y las relaciones de alteridad. Temas de Educación, 19(2), 9-26. Obtenido de http://revistas.userena.cl/index.php/ teduacion/article/view/444

Slee, R. (2011). La Escuela Extraordinaria: Exclusión, escolarización y educación inclusiva [The Irregular School: Exclusion, schooling and inclusive education] (264 ed.). Madrid [es]: Morata; Routledge.

Sosa, L. M., Acuña, A., Conese, H., \& Mirc, A. (2015). Tensiones y Paradojas sobre Discapacidad y Educación: Avances de una investigación. $11^{\circ}$ Congreso Argentino de Educación Física y Ciencias, 28 de septiembre al 10 octubre de 2015 (pág. 16). Ensenada [ar]: Universidad Nacional de La Plata. Obtenido de http://www.memoria.fahce.unlp.edu.ar/trab eventos/ ev.7362/ev.7362.pdf

Susinos Rada, T., \& Latas Parrilla, M. A. (2013). Investigación Inclusiva en Tiempos Difíciles: Certezas provisionales y debates pendientes. Reice: Revista Iberoamericana sobre Calidad Eficacia y Cambio en Educación, 11(2), 8798. Obtenido de https://revistas.uam.es/index.php/reice/article/view/2898

Thomas, G., \& Loxley, A. (2007). Deconstrucción de la Educación Especial y Construcción de la Inclusiva (200 ed.). Madrid [es]: La Muralla.

Vain, P. (2015). Las Denominaciones, Etiquetas y/o Clasificaciones como Fronteras de Exclusión Socioeducativa. Jornadas de Investigadores 2015: Fronteras y liminaridades. Espacios de diálogo, confrontación y descubrimiento (pág. 10). Posadas [ar]: Universidad Nacional de Misiones. Obtenido de http://www.comhum.com.ar/jinvestigadores/ las-denominaciones-etiquetas-yo-clasificaciones-como-fronteras-deexclusion-socioeducativa/

Venturiello, M. P. (2013). La Trama Social de la Discapacidad: Cuerpo, redes familiares y vida cotidiana (undefined ed., Vol. Ciudadanía e Inclusión). Buenos Aires [ar]: Biblos.

Zardel Jacobo, B. E. (2016). La Constitución o Producción de la Subjetividad, del Sujeto desde el Psicoanálisis y del Sujeto Sujetado al Discurso de la 'Discapacidad'. Childhood \& Philosophy, 12(24), 33. doi:10.12957/ childphilo.2016.25154

Zelmanovich, P. (2003). Contra el Desamparo. En Enseñar hoy: Una introducción a la educación en tiempos de crisis (pág. 127). Buenos Aires [ar]: Fondo de Cultura Económica. 